\title{
Global Existence and Decay for Kirchhoff Type Wave Equation with Boundary and Localized Dissipations in Exterior Domains*
}

\author{
By \\ Jeong Ja BAE \\ (Pusan National University, Korea)
}

\begin{abstract}
In this paper we prove the existence of global $\mathrm{H}^{2}$-solutions and energy decay of small amplitude of the Kirchhoff type wave equation with linear localized dissipation and two types of boundary conditions in an exterior domain. Subsequently, we also consider the same problem with weakly nonlinear dissipation.

Key Words and Phrases. Global existence of $H^{2}$-solutions, Energy decay, Exterior domain, Kirchhoff type wave equation.

2000 Mathematics Subject Classification Numbers. 35L05, 35L70, 35L20.
\end{abstract}

\section{Introduction}

In this paper, we first consider the following Kirchhoff type wave equation with two types of boundary condition:

$$
\begin{gathered}
u_{t t}-\left(1+\|\nabla u(t)\|^{2}\right) \Delta u+a(x) u_{t}=0 \quad \text { in } \Omega \times(0, \infty), \\
u=0 \quad \text { on } \Gamma_{0} \times(0, \infty), \\
\frac{\partial u}{\partial v}=-g\left(u_{t}\right) \quad \text { on } \Gamma_{1} \times(0, \infty), \\
u(x, 0)=u_{0}(x), \quad u_{t}(x, 0)=u_{1}(x),
\end{gathered}
$$

where $\Omega$ is an exterior domain in $\boldsymbol{R}^{N}$ with smooth boundary $\partial \Omega=\Gamma_{0} \cup \Gamma_{1}$, $\bar{\Gamma}_{0} \cap \bar{\Gamma}_{1}=\varnothing$.

To state precise assumption on $a(x)$, we present a set of the boundary $\Gamma_{0}$ introduced by Russell [9]:

$$
\Gamma\left(x_{0}\right)=\left\{x \in \Gamma_{0} \mid\left(x-x_{0}\right) \cdot v(x)>0\right\},
$$

where $x_{0} \in \boldsymbol{R}^{N}$ and $v(x)$ is the outward normal vector at $x \in \partial \Omega$. Note that this set is often used in control or stabilization theory for the wave equation in bounded domains (see Lions [3]). Now, we give assumptions on $a(x)$ and $g$ as follows:

\footnotetext{
* This work was supported by JSPS and KOSEF
} 
Hyp.A. There exists $x_{0} \in \boldsymbol{R}^{N}$ and a relatively open set $\omega \subset \bar{\Omega}$ such that

$$
\overline{\Gamma\left(x_{0}\right)} \subset \omega \text { and } a(x) \geq \varepsilon_{0}>0 \quad \text { for } x \in \omega \cup B_{L}^{c} \text { with some } \varepsilon_{0},
$$

where $B_{L}=\left\{x \in \boldsymbol{R}^{N}|| x \mid \leq L\right\}$.

Hyp.B. The function $g$ is in $C^{1}(\boldsymbol{R})$, strictly increasing, $g(0)=0$ and satisfies $m \leq g^{\prime}(v) \leq M$ for some positive constants $m$ and $M$.

Under the assumptions Hyp.A and Hyp.B, we prove the global existence and energy decay of small amplitude solutions for the problem (1.1).

When $\Omega$ is a bounded domain in $\boldsymbol{R}^{N}$ and $\Gamma_{0}$ is star-shaped, Lasiecka and Ong [1] are devoted to the global existence and decay estimates to the initial boundary value problem $(1.1)$ with $a(x)=0$. In [1], $g(v)$ is assumed to be a function like $g(v)=|v|^{r} v, r \geq 0$. We note that in the case of bounded domain, the boundary dissipation is sufficient for the energy decay at a certain rate, and by use of this estimate, global existence of solution is proved for a small $r>0$. One of the difficulty in the problem with Neumann boundary dissipation lies in the treatment of tangential derivatives on $\Gamma_{1}$.

On the other hand, when $\Omega$ is an exterior domain, Nakao and author [7] presented the results of decay estimate of energy $E(t)$ and related ones for the linear wave equation:

$$
\begin{gathered}
u_{t t}-\Delta u+a(x) u_{t}=0 \quad \text { in } \Omega \times(0, \infty), \\
u=0 \quad \text { on } \Gamma_{0} \times(0, \infty), \\
\frac{\partial u}{\partial v}=-g\left(u_{t}\right) \quad \text { on } \Gamma_{1} \times(0, \infty), \\
u(x, 0)=u_{0}(x), \quad u_{t}(x, 0)=u_{1}(x)
\end{gathered}
$$

under the assumption Hyp.A and Hyp.B. Our result in [7] is an extended result of Lasiecka and Triggiani [2] and Nakao [5]. In [2], Lasiecka and Triggiani treated the case where $\Omega$ is a bounded domain and $\Gamma_{0}$ is star shaped. And in [5], Nakao considered the exterior problem (1.2) for the case $\Gamma_{1}=\varnothing$.

For the Kirchhoff type quasilinear wave equation it is natural to show the existence of $H^{2}$-solutions, which means the solutions in the class $C\left([0, \infty) ; H_{2}\right) \cap$ $C^{1}\left([0, \infty) ; H_{1}\right) \cap C^{2}\left([0, \infty) ; L^{2}\right)$ or a little weaker space $L^{\infty}\left([0, \infty) ; H_{2}\right) \cap W^{1, \infty}$. $\left([0, \infty) ; H_{1}\right) \cap W^{2, \infty}\left([0, \infty) ; L^{2}\right)$.

Our first purpose of this paper is to prove the global existence and energy decay of small amplitude $H^{2}$-solutions for the Kirchhoff type wave equation (1.1) under the assumptions Hyp.A and Hyp.B. To obtain our aim, we shall derive the a priori estimates

$$
\left\|u_{t}(t)\right\|^{2}+\|\nabla u(t)\|^{2} \leq C\left(\left\|u_{0}\right\|_{H_{1}}+\left\|u_{1}\right\|_{L^{2}}\right)(1+t)^{-1}
$$


and

$$
\left\|u_{t t}(t)\right\|^{2}+\left\|\nabla u_{t}(t)\right\|^{2} \leq C\left(\left\|u_{0}\right\|_{H_{2}}+\left\|u_{1}\right\|_{H_{1}}\right)(1+t)^{-2}
$$

if $\left\|u_{0}\right\|_{H_{2}}+\left\|u_{1}\right\|_{H_{1}}$ is small. These a priori estimates are sufficient for the desired global solution. For comparison with earlier works, we mention Yamada [10] and Mochizuki [4]. Yamada [10] devoted himself to the global existence of $H^{2}$-solutions of problem (1.1) when $\Omega=\boldsymbol{R}^{N}$ and $a(x)=\lambda$ is a positive constant. Mochizuki [4] extended his result to the case of localized dissipation near infinity, that is, $a(x) \geq \varepsilon_{0}>0$ for large $|x|$.

Next, we consider the same problem with nonlinear dissipation:

$$
\begin{gathered}
u_{t t}-\left(1+\|\nabla u(t)\|^{2}\right) \Delta u+\rho\left(x, u_{t}\right)=0 \quad \text { in } \Omega \times(0, \infty), \\
u=0 \quad \text { on } \Gamma_{0} \times(0, \infty), \\
\frac{\partial u}{\partial v}=-g\left(u_{t}\right) \quad \text { on } \Gamma_{1} \times(0, \infty), \\
u(x, 0)=u_{0}(x), \quad u_{t}(x, 0)=u_{1}(x),
\end{gathered}
$$

where $\rho(x, v)$ is a nonlinear function such that $\rho_{v} \geq \varepsilon_{0}>0$ on $\omega \cup B_{L}^{c}$ for some relative open set $\omega$ and $0 \leq \rho_{v}(x, v) \leq k_{1}$ for $x \in \Omega, v \in \boldsymbol{R}$.

When $\rho\left(x, u_{t}\right)$ is nonlinear, generally no result on the decay estimate of the energy is known. But we can expect to derive the second energy decay estimate like $\left\|u_{t t}(t)\right\|^{2}+\left\|\nabla u_{t}(t)\right\|^{2} \leq C(1+t)^{-1}$ if $\left(u_{0}, u_{1}\right)$ is small. Indeed, this estimate is weaker than the case of linear dissipation, but combining this with the $L^{2}$ norm bound of $u_{t}$, Nakao and author [6] have shown the global existence of $H^{2}$-solutions for the Cauchy problem under the assumption $0<k_{0} \leq \rho^{\prime}(v) \leq$ $k_{1}\left(1+|v|^{\alpha}\right), 0 \leq \alpha \leq 2 /(N-2)^{+}(0 \leq \alpha<\infty$ if $N=1,2)$. Subsequently, in [8], we considered the global existence of $H^{2}$-solutions of the exterior problem (1.3) for the case $\Gamma_{1}=\varnothing$ by combining the ideas in [6] and [5]. Here, using these ideas, we also discuss in brief the global existence and energy decay of small amplitude solutions for the problem (1.3). We note that due to the boundary condition on $\Gamma_{1}$, our arguments become more complicate.

\section{Statement of results}

Without loss of generality we may assume $V \equiv \Omega^{c} \subset B_{L}=\left\{x \in \boldsymbol{R}^{N} \mid\right.$ $|x| \leq L\}$ and $\omega \cap \Gamma_{1}=\varnothing$. Let us introduce the following solution spaces:

$$
\begin{aligned}
X_{2}(T) \equiv & W_{l o c}^{2, \infty}\left([0, T) ; L^{2}(\Omega)\right) \cap W_{l o c}^{1, \infty}\left([0, T) ; H_{1}(\Omega)\right) \\
& \cap L_{l o c}^{\infty}\left([0, T) ; H_{2}(\Omega) \cap H_{1}(\Omega)\right),
\end{aligned}
$$




$$
\begin{gathered}
V_{1}=\left\{\left(u_{0}, u_{1}\right) \in H_{2} \cap H_{1}^{0} \times H_{1}^{0}\left|u_{0}\right|_{\Gamma_{0}}=\left.u_{1}\right|_{\Gamma_{0}}=0\right. \\
\text { and } \left.\frac{\partial u_{0}}{\partial v}=-g\left(u_{1}\right) \text { on } \Gamma_{1}\right\} .
\end{gathered}
$$

Throughout this paper, we set

$$
\begin{gathered}
E(t)=\frac{1}{2}\left[\left\|u_{t}(t)\right\|^{2}+\|\nabla u(t)\|^{2}+\frac{1}{2}\|\nabla u(t)\|^{4}\right], \\
E_{1}(t)=\frac{1}{2}\left[\left\|u_{t t}(t)\right\|^{2}+b(t)\left\|\nabla u_{t}(t)\right\|^{2}\right], \quad \text { where } b(t)=\left(1+\|\nabla u(t)\|^{2}\right) .
\end{gathered}
$$

Then our main results are as follows.

Theorem 2.1. Assume the hypotheses Hyp.A and Hyp.B hold. Then for $K>0$, there exists some open set $S_{K} \subset V_{1}$ including $(0,0)$ such that if $\left(u_{0}, u_{1}\right) \in S_{K}$, the problem (1.1) has a solution $u(t) \in X_{2} \equiv X_{2}(\infty)$ satisfying

$$
\begin{gathered}
\left\|u_{t}(t)\right\|^{2}+\|\nabla u(t)\|^{2} \leq C I_{0}^{2}(1+t)^{-1} \\
\left\|u_{t t}(t)\right\|^{2}+\left\|\nabla u_{t}(t)\right\|^{2} \leq C\left(I_{1}^{2}, K\right)(1+t)^{-2}, \\
\|\Delta u(t)\|^{2} \leq C\left(I_{1}^{2}, K\right)(1+t)^{-1} \quad \text { and } \quad \int_{0}^{t}\|\Delta u(s)\|^{2} d s \leq K^{2},
\end{gathered}
$$

where $C$ is a constant independent of $u, I_{0}=\left\|u_{0}\right\|_{H_{1}^{0}}+\left\|u_{1}\right\|_{L^{2}}$ and $I_{1}=$ $\left\|u_{0}\right\|_{H^{2}}+\left\|u_{1}\right\|_{H^{2}}$.

Next, we turn to the problem (1.3). The assumptions on $\rho(x, v)$ read as follows:

Hyp.C. $\rho(x, v)$ is measurable in $x \in \Omega$ for $v \in \boldsymbol{R}$, almost everywhere differentiable and nondecreasing function in $v$ for a.e. $x$. Further, there exist $x_{0} \in \boldsymbol{R}^{N}$ and a relatively open set $\omega \subset \bar{\Omega}$ such that

$$
\Gamma\left(x_{0}\right) \subset \omega \text { and } \rho_{v} \geq \varepsilon_{0}>0 \quad \text { for } x \in \omega \cup B_{L}^{c} \text { with some } \varepsilon_{0},
$$

where $B_{L}=\left\{x \in \boldsymbol{R}^{N}|| x \mid \leq L\right\}$. Moreover,

$$
0 \leq \rho_{v}(x, v) \leq k_{1} \quad \text { for } x \in \Omega, v \in \boldsymbol{R} .
$$

Then we can obtain more or less weaker result for the problem (1.3).

Theorem 2.2. Suppose the hypotheses Hyp.B and Hyp.C hold. Then for each $K>0$, there exists an open set $S_{K} \subset V_{1}$ including $(0,0)$ such that if $\left(u_{0}, u_{1}\right) \in S_{K}$, then the problem (1.3) admits a unique solution $u \in X_{2} \equiv X_{2}(\infty)$ satisfying 


$$
\begin{gathered}
\left\|u_{t t}(t)\right\|^{2}+\left\|\nabla u_{t}(t)\right\|^{2} \leq C\left(I_{1}^{2}, K\right)(1+t)^{-1}, \quad \int_{0}^{t}\|\Delta u(s)\|^{2} d s \leq K \\
\text { and } \quad \int_{0}^{t}\left\|\nabla u_{t}(s)\right\|^{2} d s \leq K .
\end{gathered}
$$

\section{A basic inequality}

The existence and uniqueness of local solution $u(t)$ of $(1.1)$ in $X_{2}(\tilde{T})$ for $\left(u_{0}, u_{1}\right) \in V_{1}$ is standard, see Lasiecka and Ong [1]. Here, we derive a basic differential inequality for $u(t)$. First of all, we multiply the problem (1.1) by $u_{t}$ to obtain usual energy identity:

$$
\frac{d}{d t} E(t)+\int_{\Gamma_{1}} b(t) g\left(u_{t}(t)\right) u_{t}(t) d \Gamma+\int_{\Omega} a(x)\left|u_{t}(t)\right|^{2} d x=0
$$

Let us define the functions $\phi(r), \eta(x) \in C^{1}(\bar{\Omega})$ and $h(x)=\left(h_{1}(x), \ldots, h_{N}(x)\right) \in$ $W^{1, \infty}(\bar{\Omega})$ as follows:

$$
\phi(r)= \begin{cases}\varepsilon_{0} & \text { if } 0 \leq r \leq L, \\ \varepsilon_{0} L / r & \text { if } r \geq L\end{cases}
$$

$$
\begin{gathered}
\eta(x)=1 \quad \text { on } \tilde{\omega} \cap \Omega, \quad \eta=0 \quad \text { on } \bar{\Omega} \cap \omega^{c} \quad \text { and } \quad \frac{|\nabla \eta|}{\sqrt{\eta}} \in L^{\infty}(\Omega), \\
h \cdot v \geq 0, \quad h=v \quad \text { on } \Gamma\left(x_{0}\right) \quad \text { and } \quad h(x)=0 \quad \text { on } \tilde{\omega}^{c},
\end{gathered}
$$

where $\varepsilon_{0}, L$ are the positive constants in Hyp.A, and $\tilde{\omega}$ is an open set in $\boldsymbol{R}^{N}$ such that $\overline{\Gamma\left(x_{0}\right)} \subset \tilde{\omega} \cap \bar{\Omega} \subset \omega$. Then we have the following basic differential inequality for $H^{2}$-solution $u(t)$.

Proposition 3.1. There exists constants $\varepsilon_{1}>0$ and $C>0$ such that

$$
\begin{aligned}
\frac{d}{d t} X(t) & +\varepsilon_{1} E(t)+k \int_{\Gamma_{1}} b(t) g\left(u_{t}(t)\right) u_{t}(t) d \Gamma+k \int_{\Omega} a(x)\left|u_{t}(t)\right|^{2} d x \\
\leq & C \int_{\Gamma_{1}} b(t)\left[\left|\frac{\partial u}{\partial v}\right|^{2}+\left|u_{t}(t)\right|^{2}+\left|\frac{\partial u}{\partial \tau}\right|^{2}\right] d \Gamma+C \int_{\tilde{\omega} \cap \Omega} b(t)|u(t)|^{2} d x \\
& +C \int_{\Gamma_{1}} b(t)|u(t)|^{2} d \Gamma \quad \text { for any large } k,
\end{aligned}
$$

where we set 


$$
\begin{aligned}
X(t)= & \int_{\Omega} u_{t}(t)\left[\phi\left(\left|x-x_{0}\right|\right)\left(x-x_{0}\right)-C_{0} h\right] \cdot \nabla u(t) d x+\int_{\Omega}(\alpha+\eta) u_{t}(t) u(t) d x \\
& +\frac{1}{2} \int_{\Omega}(\alpha+\eta) a(x)|u(t)|^{2} d x+k E(t) .
\end{aligned}
$$

Proof. Multiplying the problem (1.1) by $u_{t}, u, \eta(x) u, \phi\left(\left|x-x_{0}\right|\right)$. $\left(x-x_{0}\right) \cdot \nabla u$, and $h(x) \cdot \nabla u$, respectively, and combining the obtained results, then we have Proposition. In fact, the proof of this is not particularly different with the case of linear wave equation, see [7].

Now we consider the following wave equation with some special boundary conditions:

$$
\begin{gathered}
u_{t t}-\xi(t) \Delta u=0 \quad \text { in } \Omega \times[0, T], \\
u=0 \quad \text { on } \Gamma_{0} \times[0, T], \\
u=\frac{\partial u}{\partial v}=0 \quad \text { on } \Gamma_{1} \times[0, T],
\end{gathered}
$$

where $\xi(t)$ is a differentiable and uniformly positive function, say $\xi(t) \geq 1$. As a by-product of (3.2) we can prove a unique continuation property of the problem (3.3) as follows:

Corollary 3.1. Let $u \in C^{2}\left([0, T] ; L^{2}(\Omega)\right) \cap C^{1}\left([0, T] ; H_{1}^{0}(\Omega)\right) \cap C([0, T] ;$ $\left.H_{1}^{0}(\Omega) \cap H^{2}(\Omega)\right)$ be a solution of the problem (3.3). If $u_{t}(x, t)=0$ for $x \in B_{L}^{c} \cup \omega, \quad 0 \leq t \leq T$ and $\sup _{0 \leq t \leq T}\left\{\left|\xi^{\prime}(t)\right|+\left|\xi^{\prime}(t) / \xi(t)\right|\right\} \leq \delta$ with a small $\delta>0$, then there exists $T_{0}$ such that if $T>T_{0}, u \equiv 0$ on $\Omega \times[0, T]$.

Proof. To begin with, we note that $\partial u / \partial \tau=0$ on $\Gamma_{1}$ because of the fact $u=0$ on $\Gamma_{1}$. Differentiate the equation (3.3) in $t$, then $w=u_{t}$ satisfies the following problem:

$$
\begin{gathered}
w_{t t}-\xi(t) \Delta w=\xi^{\prime}(t) \Delta u\left(=-\frac{\xi^{\prime}(t)}{\xi(t)} w_{t}\right) \quad \text { in } \Omega \times[0, T], \\
w=0 \quad \text { on } \Gamma_{0} \times[0, T], \\
w=\frac{\partial w}{\partial v}=0 \quad \text { on } \Gamma_{1} \times[0, T] .
\end{gathered}
$$

It is easy to see that the solution $w=u_{t}$ of the problem (3.4) can be applicable to the inequality (3.2) in place of $u$ and we must add the terms $\left|\xi^{\prime}(t)\right|\|w\|\|\Delta u\|+\left|\xi^{\prime}(t)\right|^{2}\|\Delta u\|^{2}$ on the right hand side of corresponding inequality. Hence, using the assumption on $\xi$ and the boundary conditions, we obtain 


$$
\begin{aligned}
& \frac{d}{d t} X_{1}(t)+\varepsilon_{1} E_{1}(t) \\
& \leq C\left|\xi^{\prime}(t)\right|\left(\|\nabla w(t)\|^{2}+\|\Delta u(t)\|\left\|w_{t}(t)\right\|+\|\Delta u(t)\|\left\|u_{t}(t)\right\|\right. \\
& \left.+\|\Delta u(t)\|\|\nabla w(t)\|+\left|\xi^{\prime}(t)\right|\|\Delta u(t)\|^{2}\right) \\
& \leq C\left(\left|\xi^{\prime}(t)\right|\|\nabla w(t)\|^{2}+\left|\frac{\xi^{\prime}(t)}{\xi(t)}\right|\left\|u_{t t}(t)\right\|\left[\left\|w_{t}(t)\right\|+\left\|u_{t}(t)\right\|+\|\nabla w(t)\|\right]\right) \\
& \quad+C\left|\frac{\xi^{\prime}(t)}{\xi(t)}\right|^{2}\left\|w_{t}(t)\right\|^{2} \\
& \leq C_{1} \delta E_{1}(t), \quad 0 \leq t \leq T,
\end{aligned}
$$

where

$$
\begin{aligned}
X_{1}(t)= & \int_{\Omega} w_{t}(t)\left[\phi\left(\left|x-x_{0}\right|\right)\left(x-x_{0}\right)-C_{0} h\right] \cdot \nabla w(t) d x \\
& +\int_{\Omega}(\alpha+\eta) w_{t}(t) w(t) d x+k E_{1}(t)
\end{aligned}
$$

and $E_{1}(t)=\left[\left\|w_{t}(t)\right\|^{2}+\xi(t)\|\nabla w(t)\|^{2}\right] / 2$. Thus for small $\delta>0$, we have

$$
X_{1}(T)+\varepsilon_{1} \int_{0}^{T} E_{1}(t) d t \leq X_{1}(0)+C_{1} \delta \int_{0}^{T} E_{1}(t) d t .
$$

Using the fact that $X_{1}(t)$ is equivalent to $E_{1}(t)$ for large $k>0$, we get

$$
E_{1}(T)+\widetilde{\varepsilon}_{1} \int_{0}^{T} E_{1}(t) d t \leq C_{2} E_{1}(0)+C_{1} \delta \int_{0}^{T} E_{1}(t) d t .
$$

On the other hand, let $E_{1}\left(t^{*}\right)=\min _{0 \leq t \leq T} E_{1}(t), 0 \leq t^{*} \leq T$, then we have from (3.4) that for $0 \leq t \leq T$,

$$
\begin{aligned}
E_{1}(t) & =E_{1}\left(t^{*}\right)+\frac{1}{2} \int_{t^{*}}^{t} \xi^{\prime}(s)\|\nabla w(s)\|^{2} d s+\int_{t^{*}}^{t} \int_{\Omega} \xi^{\prime}(s) \Delta u(s) w_{t}(s) d x d s \\
& =E_{1}\left(t^{*}\right)+\frac{1}{2} \int_{t^{*}}^{t} \xi^{\prime}(s)\|\nabla w(s)\|^{2} d s+\int_{t^{*}}^{t} \frac{\xi^{\prime}(s)}{\xi(s)}\left\|w_{t}(s)\right\|^{2} d s
\end{aligned}
$$

and so

$$
E_{1}(t) \leq E_{1}\left(t^{*}\right)+C_{3} \delta \int_{0}^{T} E_{1}(s) d s \quad \text { for all } 0 \leq t \leq T .
$$

We use the last inequality for $t=0$. Then, if $\delta<\tilde{\varepsilon}_{1} / C_{4}$ with some $C_{4}=$ $C_{1}+C_{2} C_{3}>0$, we get 


$$
T E_{1}\left(t^{*}\right) \leq \int_{0}^{T} E_{1}(t) d t \leq C_{5} E_{1}\left(t^{*}\right)
$$

for some constant $C_{5}=C_{2} /\left(\tilde{\varepsilon}_{1}-C_{4} \delta\right)>0$. Taking $T>C_{5} \equiv T_{0}$, we see $E_{1}\left(t^{*}\right)=0$ i.e., $w_{t}\left(t^{*}\right)=\nabla w\left(t^{*}\right)=0$. Considering again equation (3.4), we can see that $w(t)=u_{t}(t) \equiv 0$, and so the original problem (3.3) implies

$$
\Delta u=0 \quad \text { on } \Omega \times[0, T] .
$$

Thus we conclude by boundary conditions

$$
u(x, t) \equiv 0 \quad \text { on } \Omega \times[0, T] .
$$

\section{Energy decay}

We continue the estimation of the local solutions $u$ in $X_{2}(\tilde{T})$. Let $\varepsilon_{2}$, $K>0$ and we assume for a moment

$$
E(t) \leq \varepsilon_{2} \quad \text { and } \quad\left\|\nabla u_{t}(t)\right\| \leq K(1+t)^{-1}, \quad 0 \leq t<\tilde{T} .
$$

Now, we have to estimate the integrals $\int_{\tilde{\omega} \cap \Omega} b(t)|u(t)|^{2} d x+\int_{\Gamma_{1}} b(t)|u(t)|^{2} d \Gamma$ in the right hand side of (3.2).

Proposition 4.1. Let $u$ be a solution of (1.1). In addition to the assumption (4.1), we assume that

$$
K\|\nabla u(t)\| \leq \delta
$$

with small $\delta>0$. Then there exists $T_{0}>0$ such that if $T>T_{0}$, the solution $u$ satisfies

$$
\begin{aligned}
& \int_{t}^{t+T} \int_{\Omega_{L}}|u(s)|^{2} d x d s+\int_{t}^{t+T} \int_{\Gamma_{1}}|u(s)|^{2} d \Gamma d s \\
& \leq C_{0}\left\{\int_{t}^{t+T} \int_{\Omega} a(x)\left|u_{t}(s)\right|^{2} d x d s+\int_{t}^{t+T} \int_{\Gamma_{1}}\left[\left|\frac{\partial u}{\partial v}\right|^{2}+\left|u_{t}(s)\right|^{2}\right] d \Gamma d s\right\} \\
& \quad+\varepsilon \int_{t}^{t+T} E(s) d s, \quad 0 \leq t<\tilde{T}-T
\end{aligned}
$$

where $C_{0}=C_{0}\left(T, K, \varepsilon_{2}\right)$ is a constant independent of $u$.

Proof. We can prove Proposition using contradiction method as in [7] and [1]. Assume that (4.3) does not hold, there would exist sequences of numbers $\left\{t_{n}\right\}$ and solutions $\left\{u_{n}\right\}$, respectively, such that 


$$
\begin{aligned}
\int_{t_{n}}^{t_{n}+T} & \int_{\Omega_{L}}\left|u_{n}(t)\right|^{2} d x d t+\int_{t_{n}}^{t_{n}+T} \int_{\Gamma_{1}}\left|u_{n}(t)\right|^{2} d \Gamma d t \\
\geq & n\left\{\int_{t_{n}}^{t_{n}+T}\left[\int_{\Omega} a(x)\left|u_{n t}(t)\right|^{2} d x+\int_{\Gamma_{1}}\left(\left|\frac{\partial u_{n}}{\partial v}(t)\right|^{2}+\left|u_{n t}(t)\right|^{2}\right) d \Gamma\right] d t\right\} \\
& +\varepsilon \int_{t_{n}}^{t_{n}+T} E_{n}(t) d t
\end{aligned}
$$

where $E_{n}(t)$ is defined by $E(t)$ with $u$ replaced by $u_{n}$.

Set $\lambda_{n}^{2}=\int_{t_{n}}^{t_{n}+T} \int_{\Omega_{L}}\left|u_{n}(t)\right|^{2} d x d t+\int_{t_{n}}^{t_{n}+T} \int_{\Gamma_{1}}\left|u_{n}(t)\right|^{2} d \Gamma d t$ and $v_{n}(t)=u_{n}\left(t+t_{n}\right) /$ $\lambda_{n}$. Then we can derive

$$
\begin{gathered}
\int_{0}^{T} \int_{\Omega} a(x)\left|v_{n t}(t)\right|^{2} d x d t+\int_{0}^{T} \int_{\Gamma_{1}}\left(\left|\frac{\partial v_{n}}{\partial v}(t)\right|^{2}+\left|v_{n t}(t)\right|^{2}\right) d \Gamma d t \rightarrow 0 \quad \text { as } n \rightarrow \infty \\
\int_{0}^{T} \int_{\Omega}\left(\left|v_{n t}(t)\right|^{2}+\left|\nabla v_{n}(t)\right|^{2}\right) d x \leq \frac{2}{\lambda_{n}^{2}} \int_{t_{n}}^{t_{n}+T} E_{n}(t) d t \leq \frac{2}{\varepsilon}<\infty \\
\int_{0}^{T} \int_{\Omega_{L}}\left|v_{n}(t)\right|^{2} d x d t+\int_{0}^{T} \int_{\Gamma_{1}}\left|v_{n}(t)\right|^{2} d \Gamma d t=1 .
\end{gathered}
$$

Thus there exists a subsequence of $v_{n}(t)$ (same symbol) such that $v_{n}(t)$ converges weakly to a function $v \in L^{2}\left(0, T ; L_{l o c}^{2}(\Omega)\right)$ such that $v_{t} \in L^{2}\left(0, T ; L^{2}(\Omega)\right) \cap$ $L^{2}\left(0, T ; L^{2}\left(\Gamma_{1}\right)\right), \nabla v \in L^{2}\left(0, T ; L^{2}(\Omega)\right)$. By Rellich's lemma, we may assume that $v_{n}(t)$ converges to $v(t)$ strongly in $L^{2}\left(\Omega_{L} \times[0, T]\right) \cap L^{2}\left(\Gamma_{1} \times[0, T]\right)$. On the other hand, taking into account the assumption (4.1) and Ascoli-Arzela Theorem, there exists a subsequence $u_{n}(t)$ (same symbol) such that

$$
\lim _{n \rightarrow \infty}\left\|\nabla u_{n}(t)\right\| \rightarrow \lambda(t) \quad \text { in } C([0, T]) .
$$

Hence the limit function $v(t)$ satisfies

$$
\int_{0}^{T} \int_{\Omega_{L}}|v(t)|^{2} d x d t+\int_{0}^{T} \int_{\Gamma_{1}}|v(t)|^{2} d \Gamma d t=1
$$

and

$$
\begin{gathered}
v_{t t}(t)-\left(1+\lambda(t)^{2}\right) \Delta v(t)=0 \quad \text { on } \Omega \times[0, T], \\
v=0 \quad \text { on } \Gamma_{0} \times(0, \infty), \\
v_{t}=0, \quad \frac{\partial v}{\partial v}=0 \quad \text { on } \Gamma_{1} \times(0, \infty) .
\end{gathered}
$$

Since $a(x) \geq \varepsilon_{0}>0$ on $B_{L}^{c} \cup \omega$ and $\int_{0}^{T} \int_{\Omega} a(x)\left|v_{n t}(s)\right|^{2} d x d s \rightarrow 0$ as $n \rightarrow \infty$,

$$
v_{t}(x, t)=0 \quad \text { on }\left(B_{L}^{c} \cup \omega\right) \times[0, T] .
$$


We also note that the assumptions (4.1)-(4.2) imply

$$
\begin{aligned}
\frac{1}{1+\lambda(t)^{2}} \frac{d}{d t}\left|1+\lambda(t)^{2}\right| \leq \frac{d}{d t}\left|1+\lambda(t)^{2}\right| & \leq \lim _{n \rightarrow \infty}\left|\frac{d}{d t}\left(1+\left\|\nabla u_{n}(t)\right\|^{2}\right)\right| \\
& =2 \lim _{n \rightarrow \infty}\left|\left(\nabla u_{n}(t), \nabla u_{n t}(t)\right)\right| \\
& \leq 2 K\left\|\nabla u_{n}(t)\right\| \leq 2 \delta .
\end{aligned}
$$

Applying Corollary 3.1, we see that if $T>T_{0}$,

$$
v_{t}(x, t)=0 \quad \text { on } \Omega \times[0, T] .
$$

The fact (4.6) means that $v(x, t)=v(x)$ is independent of $t$. Thus by (4.5), we have $-\Delta v=0$ on $\Omega \times[0, T]$. Since $\nabla v \in L^{2}(\Omega)$ and $\left.v\right|_{\Gamma_{0}}=\partial u /\left.\partial v\right|_{\Gamma_{1}}=0$, we conclude $v(x) \equiv 0$ in $\Omega$. This contradicts to (4.4).

Now, we take $T$ with $T_{0} \leq T<2 T_{0}$ and a natural number $n \in N$ with $n T \leq t<(n+1) T$. Applying Proposition 4.1 on the interval $[t-(i+1) T$, $t-i T], i=0,1, \ldots, n-1$, then for all $i=0,1, \ldots, n-1$, we have $n$-inequalities $\int_{t-(i+1) T}^{t-i T} \int_{\Omega_{L}}|u(s)|^{2} d x d s+\int_{t-(i+1) T}^{t-i T} \int_{\Gamma_{1}}|u(s)|^{2} d \Gamma d s$. Combining them, we have the following inequality independent on $T$ in the interval of integration, which is an essential tool in proving our argument.

Proposition 4.2. Let $u(t)$ be a solution of (1.1). Under the assumptions (4.1) and (4.2), we have

$$
\begin{aligned}
\int_{0}^{t} \int_{\Omega_{L}} & |u(s)|^{2} d x d s+\int_{0}^{t} \int_{\Gamma_{1}}|u(s)|^{2} d \Gamma d s \\
\leq & C_{\varepsilon}\left\{\int_{0}^{t} \int_{\Omega} a(x)\left|u_{t}(s)\right|^{2} d x d s+\int_{0}^{t} \int_{\Gamma_{1}}\left\{\left|\frac{\partial u}{\partial v}(s)\right|^{2}+\left|u_{t}(s)\right|^{2}\right\} d \Gamma d s\right\} \\
& +\varepsilon \int_{0}^{t} E(s) d s
\end{aligned}
$$

for any $t \geq T_{0}$ and $0<\varepsilon \ll 1$, where $C_{\varepsilon}$ is a constant independent of $t$ and $u$.

Applying the inequality in Proposition 3.1 along with the ones in Proposition 4.1 and Proposition 4.2, respectively, we have the followings.

Proposition 4.3. Let $u$ be a solution of the problem (1.1). Then for large $k>0$ and $T>T_{0}$ fixed, if $0<t+T<\tilde{T}$, we have

$$
\begin{aligned}
X(t+ & T)-X(t)+\frac{\varepsilon_{1}}{2} \int_{t}^{t+T} E(s) d s \\
& \leq C_{\varepsilon} \int_{t}^{t+T} \int_{\Gamma_{1}} b(s)\left[\left|\frac{\partial u}{\partial v}\right|^{2}+\left|u_{t}(s)\right|^{2}+\left|\frac{\partial u}{\partial \tau}\right|^{2}\right] d \Gamma d s
\end{aligned}
$$


and

$$
\begin{aligned}
X(t) & +\varepsilon_{1} \int_{0}^{t} E(s) d s \\
& \leq C \int_{0}^{t} \int_{\Gamma_{1}} b(s)\left[\left|\frac{\partial u}{\partial v}\right|^{2}+\left|u_{t}(s)\right|^{2}+\left|\frac{\partial u}{\partial \tau}\right|^{2}\right] d \Gamma d s+C X(0), \quad t \geq 0
\end{aligned}
$$

with some constant $C>0$ independent of $t$ and $u$.

The second inequality (4.9) is proved by using (4.7) and the following two inequalities:

$$
m \int_{0}^{t} \int_{\Gamma_{1}}\left|u_{t}(s)\right|^{2} d \Gamma d s \leq \int_{0}^{t} \int_{\Gamma_{1}} g\left(u_{t}(s)\right) u_{t}(s) d \Gamma d s \leq C E(0)
$$

and

$$
\int_{0}^{t} \int_{\Omega} a(x)\left|u_{t}(s)\right|^{2} d x d s \leq C E(0) .
$$

Now, to control the tangential derivatives in the inequality (4.9), we use Lasiecka and Ong's result [1]. But they estimated the size of tangential derivative in bounded domain, so we have to restrict our problem to the ones in bounded domain. For this reason as in [7], we take a function $\psi(x) \in C^{1}(\bar{\Omega})$ such that $\psi(x)=1$ in a neighborhood of $\Gamma_{1}$ and $\psi(x)=0$ for $|x| \geq L$ and $w(x, t)=\psi(x) u$. Then we have

$$
\begin{gathered}
w_{t t}-b(t) \Delta w=-a(x) w_{t}-2 b(t) \nabla \psi(x) \cdot \nabla u-b(t) \Delta \psi u \\
\equiv f(x, t) \quad \text { in } \Omega_{L} \times[0, \infty), \\
\left.w\right|_{\Gamma_{0}}=\left.w\right|_{\partial B_{L}}=0
\end{gathered}
$$

and we can use the following Lasiecka and Ong's result in bounded domain by the condition of $\psi$.

Proposition 4.4 (Lasiecka and Ong [1]). We fix $T>0$. Let $t \geq T>0$ and let $u$ be a solution in Theorem 2.1. Then for any constant $\varepsilon_{0}$ with $0<\varepsilon_{0}<1 / 2$ the following trace estimate holds

$$
\begin{aligned}
& \int_{t-3 T / 4}^{t-T / 4} \int_{\Gamma_{1}}\left|\frac{\partial u}{\partial \tau}\right|^{2} d \Gamma d s \\
& \leq C \int_{t-T}^{t}\left[\int_{\Gamma_{1}}\left(\left|\frac{\partial u}{\partial v}\right|^{2}+\left|u_{t}(s)\right|^{2}\right) d \Gamma+\|f(s)\|_{H^{-1 / 2+\varepsilon_{0}}\left(\Omega_{L}\right)}^{2}\right] d s \\
& \quad+C_{\varepsilon_{0}}\|w\|_{H^{1 / 2+\varepsilon_{0}}\left(Q_{L, T, t}\right)}^{2}
\end{aligned}
$$


where we set $Q_{L, T, t}=\Omega_{L} \times[t-T, t]$. The constants $C, C_{\varepsilon_{0}}$ in the above are independent of $t$ and $u$.

Remark. In [1], Lasiecka and Ong treated the size of the tangential derivative in $\Gamma_{1} \times[\delta, t-\delta], \delta>0$, we apply their result for $t \geq T>0, t-T$ and $T / 4$ in place of 0 and $\delta$, respectively.

It is easy to see that as in [7]

$$
\begin{gathered}
\int_{t-T}^{t}\|f(s)\|_{H^{-1 / 2+\varepsilon_{0}\left(\Omega_{L}\right)}}^{2} d s \leq C \int_{t-T}^{t}\left\|a(x) u_{t}(s)\right\|_{L^{2}\left(\Omega_{L}\right)}^{2} d s+C_{\varepsilon_{0}}\|u\|_{H^{1 / 2+\varepsilon_{0}}\left(Q_{L, T, t}\right)}^{2}, \\
\|w\|_{H^{1 / 2+\varepsilon_{0}}\left(Q_{L, T}\right)}^{2} \leq C\|u\|_{H^{1 / 2+\varepsilon_{0}}\left(Q_{L, T, t}\right)}^{2}
\end{gathered}
$$

Reiterating the inequality (4.12) to $t=i T / 2, i=2,3, \ldots, 2 n$ with $n T \leq t<$ $(n+1) T$, summing up the resulted inequalities and then combining with (4.13), we have

$$
\begin{aligned}
\int_{T / 4}^{t-T / 4} \int_{\Gamma_{1}}\left|\frac{\partial u}{\partial \tau}\right|^{2} d \Gamma d s \\
\leq C \int_{0}^{t}\left[\int_{\Gamma_{1}}\left(\left|\frac{\partial u}{\partial v}\right|^{2}+\left|u_{t}(s)\right|^{2}\right) d \Gamma+\left\|a(x) u_{t}(s)\right\|_{L^{2}\left(\Omega_{L}\right)}^{2}\right] d s \\
\quad+C_{\varepsilon_{0}}\|u\|_{H^{1 / 2+\varepsilon_{0}}\left(Q_{L, t}\right)}^{2}
\end{aligned}
$$

Using the interpolation theory, Proposition 4.2 and the boundary condition on $\Gamma_{1}$, we get

$$
\begin{aligned}
C\|u\|_{H^{1 / 2+\varepsilon_{0}}\left(Q_{L, t}\right)}^{2} & \leq \frac{\varepsilon_{1}}{4} \int_{0}^{t}\left(\|u(s)\|_{H^{1}\left(\Omega_{L}\right)}^{2}+\left\|u_{t}(s)\right\|^{2}\right) d s+C \int_{0}^{t} \int_{\Omega_{L}}|u(s)|^{2} d x d s \\
& \leq C E(0)+\frac{\varepsilon_{1}}{2} \int_{0}^{t} E(s) d s .
\end{aligned}
$$

Applying Proposition 4.3 on $[T / 4, t-T / 4]$ in place of $[t, t+T]$, and then combining the estimates (4.14), (4.15) and the inequality $E(t) \leq E(0)$ for $t \geq 0$, we have

$$
X(t-T / 4)+\frac{\varepsilon_{1}}{2} \int_{0}^{t} E(s) d s \leq C(X(0)+E(0)) .
$$

Using the equivalence relation of $X(t)$ with $E(t)+\|u(t)\|^{2}$ and the fact $\|u(t)\|^{2} \leq \int_{t-T / 4}^{t}\left\|u_{t}(s)\right\|^{2} d s+\|u(t-T / 4)\|^{2}$, we can get $X(t) \leq C X(t-T / 4)$ for $t \geq T / 4$. Thus we have 


$$
X(t)+\tilde{\varepsilon}_{1} \int_{0}^{t} E(s) d s \leq C X(0) \quad \text { for some } \tilde{\varepsilon}_{1}>0
$$

or

$$
\sup _{0 \leq t<\infty} X(t)+\tilde{\varepsilon}_{1} \int_{0}^{\infty} E(s) d s \leq C X(0)
$$

By (3.1), we have

$$
\frac{d}{d t}[(1+t) E(t)]=E(t)+(1+t) \frac{d}{d t} E(t) \leq E(t) .
$$

Integrating (4.19) over $(0, t)$, we obtain

$$
(1+t) E(t) \leq \int_{0}^{t} E(s) d s+E(0) \leq C X(0)
$$

and so we can conclude

$$
E(t) \leq C X(0)(1+t)^{-1}
$$

\section{Estimates for the second order derivatives and proof of Theorem 2.1}

To end the proof of Theorem 2.1 we remain to derive some estimates for the second order derivatives of a local solution $u(t)$ satisfying (4.1). Note that $w=u_{t}$ satisfies the following linear equation:

$$
\begin{gathered}
w_{t t}-b(t) \Delta w-b^{\prime}(t) \Delta u+a(x) w_{t}=0 \quad \text { in } \Omega \times(0, \infty), \\
w=0 \quad \text { on } \Gamma_{0} \times(0, \infty), \\
\frac{\partial w}{\partial v}=-g^{\prime}\left(u_{t}\right) w_{t} \quad \text { on } \Gamma_{1} \times(0, \infty), \\
w(0)=u_{1}, \quad w_{t}(0)=b(0) \Delta u_{0}-a(x) u_{1} \quad \text { in } \Omega .
\end{gathered}
$$

First of all, we have the following energy identity

$$
\begin{gathered}
\frac{d}{d t} E_{1}(t)+\int_{\Gamma_{1}} b(t) g^{\prime}\left(u_{t}(t)\right)\left|w_{t}(t)\right|^{2} d \Gamma+\int_{\Omega} a(x)\left|w_{t}(t)\right|^{2} d x \\
=\frac{1}{2} \int_{\Omega} b^{\prime}(t)|\nabla w(t)|^{2} d x+\int_{\Omega} b^{\prime}(t) \Delta u(t) w_{t}(t) d x .
\end{gathered}
$$


Applying the usual multiplier method as section 3, we get

$$
\begin{aligned}
& \frac{d}{d t} X_{1}(t)+\varepsilon_{1} E_{1}(t)+k \int_{\Gamma_{1}} b(t) g^{\prime}(w(t))\left|w_{t}(t)\right|^{2} d \Gamma+\int_{\Omega} a(x)\left|w_{t}(t)\right|^{2} d x \\
& \leq C \int_{\Gamma_{1}} b(t)\left[\left|\frac{\partial w}{\partial v}\right|^{2}+\left|w_{t}(s)\right|^{2}+\left|\frac{\partial w}{\partial \tau}\right|^{2}\right] d \Gamma \\
& \quad+C \int_{\Omega}\left|b^{\prime}(t)\right|\left(\left|w_{t}(t)\right|^{2}+|\nabla w(t)|^{2}+|\Delta u(t)|^{2}+|w(t)|^{2}\right. \\
& \left.\quad+\left|b^{\prime}(t)\right||\Delta u(t)|^{2}\right) d x \\
& \quad+C \int_{\tilde{\omega} \cap \Omega} b(t)|w(t)|^{2} d x+C \int_{\Gamma_{1}} b(t)|w(t)|^{2} d \Gamma
\end{aligned}
$$

where

$$
\begin{aligned}
X_{1}(t)= & \int_{\Omega} w_{t}(t)\left[\phi\left(\left|x-x_{0}\right|\right)\left(x-x_{0}\right)-C_{0} h\right] \cdot \nabla w(t) d x \\
& +\int_{\Omega}(\alpha+\eta) w_{t}(t) w(t) d x+\frac{1}{2} \int_{\Omega}(\alpha+\eta) a(x)|w(t)|^{2} d x+k E_{1}(t) .
\end{aligned}
$$

Also we treat integral $\int_{\tilde{\omega} \cap \Omega}|w(t)|^{2} d x+\int_{\Gamma_{1}}|w(t)|^{2} d \Gamma$ using unique continuation principle as section 4 .

Proposition 5.1. Let $u$ be a solution of (1.1). Then under the assumptions (4.1) and (4.2), there exists $T_{0}>0$ such that if $T>T_{0}, w=u_{t}$ satisfies

$$
\begin{aligned}
& \int_{t}^{t+T} \int_{\Omega_{L}}|w(s)|^{2} d x d s+\int_{t}^{t+T} \int_{\Gamma_{1}}|w(s)|^{2} d \Gamma d s \\
& \leq C\left\{\int_{t}^{t+T} \int_{\Omega} a(x)\left|w_{t}(s)\right|^{2} d x d s+\int_{t}^{t+T} \int_{\Gamma_{1}}\left[\left|\frac{\partial w}{\partial v}\right|^{2}+\left|w_{t}(s)\right|^{2}\right] d \Gamma d s\right\} \\
&+ \int_{t}^{t+T} \int_{\Omega}\left|b^{\prime}(s)\right|^{2}|\Delta u(s)|^{2} d x d s+\varepsilon \int_{t}^{t+T} E_{1}(s) d s
\end{aligned}
$$

where $C=C\left(T, K, \varepsilon_{2}\right)$ is a constant independent of $u$.

Reiterating the argument obtaining (4.8) and (4.9), we can derive for sufficiently small $\varepsilon>0$ 


$$
\begin{aligned}
X_{1}(t+ & T)-X_{1}(t)+\varepsilon_{1} \int_{t}^{t+T} E_{1}(s) d s \\
\leq & C \int_{t}^{t+T} \int_{\Gamma_{1}} b(s)\left[\left|\frac{\partial w}{\partial v}\right|^{2}+\left|w_{t}(s)\right|^{2}+\left|\frac{\partial w}{\partial \tau}\right|^{2}\right] d \Gamma d s \\
& +C \int_{t}^{t+T} \int_{\Omega}\left|b^{\prime}(s)\right|\left(\left|w_{t}(s)\right|^{2}+|\nabla w(s)|^{2}+|\Delta u(s)|^{2}+|w(s)|^{2}\right) d x d s \\
& +C \int_{t}^{t+T} \int_{\Omega}\left|b^{\prime}(s)\right|^{2}|\Delta u(s)|^{2} d x d s
\end{aligned}
$$

and

$$
\begin{aligned}
X_{1}(t)+ & \varepsilon_{1} \int_{0}^{t} E_{1}(s) d s \\
\leq & C X_{1}(0)+C \int_{0}^{t} \int_{\Gamma_{1}} b(s)\left[\left|\frac{\partial w}{\partial v}\right|^{2}+\left|w_{t}(s)\right|^{2}+\left|\frac{\partial w}{\partial \tau}\right|^{2}\right] d \Gamma d s \\
& +C \int_{0}^{t} \int_{\Omega}\left|b^{\prime}(s)\right|\left(\left|w_{t}(s)\right|^{2}+|\nabla w(s)|^{2}+|\Delta u(s)|^{2}+|w(s)|^{2}\right) d x d s \\
& +C \int_{0}^{t} \int_{\Omega}\left|b^{\prime}(s)\right|^{2}|\Delta u(s)|^{2} d x d s .
\end{aligned}
$$

Applying Proposition 4.4 to $w=u_{t}$ in place of $u$ and reiterating the argument obtaining (4.17), we get the following inequality

$$
\begin{aligned}
X_{1}(t)+ & \varepsilon_{1} \int_{0}^{t} E_{1}(s) d s \\
\leq & C X_{1}(0)+C \int_{0}^{t} \int_{\Omega}\left|b^{\prime}(s)\right|\left(\left|w_{t}(s)\right|^{2}+|\nabla w(s)|^{2}+|\Delta u(s)|^{2}\right. \\
& \left.+|w(s)|^{2}\right) d x d s+C \int_{0}^{t} \int_{\Omega}\left|b^{\prime}(s)\right|^{2}|\Delta u(s)|^{2} d x d s .
\end{aligned}
$$

To estimate the integrals in the right hand side of (5.7), we assume for a moment

$$
\begin{gathered}
E(t) \leq \varepsilon_{2}, \quad E_{1}(t) \leq K^{2}(1+t)^{-2}, \quad\|\Delta u(t)\|^{2} \leq K^{2}(1+t)^{-1} \\
\text { and } \quad \int_{0}^{t}\|\Delta u(s)\|^{2} d s \leq K^{2} .
\end{gathered}
$$

Using the fact that 


$$
E(t) \leq C X(0)(1+t)^{-1}
$$

and the preassumption (5.8), we have the following Proposition.

Proposition 5.2. Under the assumption (5.8), we have

$$
\begin{aligned}
& \int_{0}^{\tilde{T}} \int_{\Omega}\left|b^{\prime}(s)\right|\left(\left|w_{t}(s)\right|^{2}+|\nabla w(s)|^{2}+|\Delta u(s)|^{2}+|w(s)|^{2}+\left|b^{\prime}(s)\right||\Delta u(s)|^{2}\right) d x d s \\
& \quad \leq C\left[K^{3} E(0)^{1 / 2}+K E(0)^{1 / 2} X(0)+K^{4} E(0)\right] \\
& \quad \equiv q(K, E(0))
\end{aligned}
$$

where $\lim _{E(0) \rightarrow 0} q(K, E(0))=0$.

Using this result, we derive the following result.

Proposition 5.3. Under the assumption (5.8), we can deduce

$$
\begin{gathered}
X_{1}(t)+C \int_{0}^{\tilde{T}} E_{1}(s) d s \leq C X_{1}(0)+q(K, E(0)), \\
k \int_{0}^{\tilde{T}} \int_{\Omega} a(x)\left|w_{t}(s)\right|^{2} d x d s+k \int_{0}^{\tilde{T}} \int_{\Gamma_{1}} b(s)\left|w_{t}(s)\right|^{2} d \Gamma d s \\
\leq C X_{1}(0)+q(K, E(0)), \\
\int_{0}^{\tilde{T}}\|w(t)\|^{2} d t \leq \frac{1}{\varepsilon_{0}} E(0)+C X_{1}(0)+q(K, E(0)) \\
\equiv q_{0}\left(K, E_{1}(0), E(0)\right) .
\end{gathered}
$$

Proof. Using (5.7), (5.10) and (5.2), we easily obtain (5.11) and (5.12). Next, from (5.12), the assumption on $g$ and boundary condition, we have

$$
k \int_{0}^{t} \int_{\Gamma_{1}}\left(\left|w_{t}(s)\right|^{2}+\left|\frac{\partial w}{\partial v}\right|^{2}\right) d \Gamma d s \leq C X_{1}(0)+q(K, E(0)) .
$$

Thus (5.11), (5.12) and (5.14) imply

$$
\begin{aligned}
\int_{0}^{\tilde{T}}\|w(t)\|^{2} d t & =\int_{0}^{\tilde{T}} \int_{B_{L}^{c}}|w(t)|^{2} d x d t+\int_{0}^{\tilde{T}} \int_{\Omega_{L}}|w(t)|^{2} d x d t \\
& \leq \frac{1}{\varepsilon_{0}} E(0)+C X_{1}(0)+q(K, E(0)) \equiv q_{0}\left(K, E_{1}(0), E(0)\right) .
\end{aligned}
$$

Note that $\lim _{E(0), E_{1}(0) \rightarrow 0} q_{0}\left(K, E_{1}(0), E(0)\right)=0$. 
Next we shall show

$$
\int_{0}^{\tilde{T}}(1+t) E_{1}(t) d t<\infty .
$$

Now, the equation (5.2) yields

$$
\begin{aligned}
\frac{d}{d t}\left[(1+t) E_{1}(t)\right]= & E_{1}(t)+(1+t) \frac{d}{d t} E_{1}(t) \\
\leq & E_{1}(t)-(1+t)\left[b(t) \int_{\Gamma_{1}} g^{\prime}\left(u_{t}(t)\right)\left|w_{t}(t)\right|^{2} d \Gamma+\int_{\Omega} a(x)\left|w_{t}(t)\right|^{2} d x\right] \\
& +C(1+t) \int_{\Omega}\left|b^{\prime}(t)\right|\left[|\Delta u(t)|^{2}+\left|w_{t}(t)\right|^{2}+|\nabla w(t)|^{2}\right] d x .
\end{aligned}
$$

Integrating the above inequality, (5.11) implies

$$
\begin{aligned}
(1+t) & E_{1}(t)+\int_{0}^{t}(1+s)\left[b(s) \int_{\Gamma_{1}} g^{\prime}\left(u_{t}(s)\right)\left|w_{t}(s)\right|^{2} d \Gamma+\int_{\Omega} a(x)\left|w_{t}(s)\right|^{2} d x\right] d s \\
\leq & C X_{1}(0)+C E_{1}(0)+q(K, E(0)) \\
& +C \int_{0}^{t}(1+s) \int_{\Omega}\left|b^{\prime}(s)\right|\left[|\Delta u(s)|^{2}+\left|w_{t}(s)\right|^{2}+|\nabla w(s)|^{2}\right] d x d s .
\end{aligned}
$$

Substitute $\sqrt{1+s} w$ in replace of $w$ in Proposition 5.1, we can get from (5.16),

$$
\begin{aligned}
\int_{t}^{t+T} \int_{\Omega_{L}}(1+s)|w(s)|^{2} d x d s \\
\leq C_{\varepsilon} \int_{t}^{t+T}(1+s)\left\{\int_{\Omega} a(x)\left|w_{t}(s)\right|^{2} d x+\int_{\Gamma_{1}}\left[\left|w_{t}(s)\right|^{2}+\left|\frac{\partial w}{\partial v}\right|^{2}\right] d \Gamma\right\} d s \\
\quad+C_{\varepsilon} \int_{t}^{t+T} \int_{\Omega}(1+s)\left|b^{\prime}(s)\right|^{2}|\Delta u(s)|^{2} d x d s+\varepsilon \int_{t}^{t+T}(1+s) E_{1}(s) d s \\
\leq C X_{1}(0)+C E_{1}(0)+q(K, E(0))+\varepsilon \int_{0}^{t}(1+s) E_{1}(s) d s \\
\quad+C \int_{0}^{t}(1+s) \int_{\Omega}\left|b^{\prime}(s)\right|\left[|\Delta u(s)|^{2}+\left|w_{t}(s)\right|^{2}+|\nabla w(s)|^{2}\right] d x d s \\
\quad+C \int_{0}^{t}(1+s) \int_{\Omega}\left|b^{\prime}(s)\right|^{2}|\Delta u(s)|^{2} d x d s .
\end{aligned}
$$

We multiply $(5.5)$ by $(1+t+T)$, we can show for any $t \geq 0$, 


$$
\begin{aligned}
& (1+t) X_{1}(t)+\varepsilon_{1} \int_{0}^{t} E_{1}(s) d s \\
& \leq(1+T) \sup _{0 \leq s \leq T} X_{1}(s)+\sum_{i=1}^{n} T X_{1}(t-i T) \\
& \quad+C \int_{0}^{t}(1+s) \int_{\Gamma_{1}} b(s)\left[\left|\frac{\partial w}{\partial v}\right|^{2}+\left|w_{t}(s)\right|^{2}+\left|\frac{\partial w}{\partial \tau}\right|^{2}\right] d \Gamma d s \\
& \quad+C \int_{0}^{t}(1+s) \int_{\Omega}\left|b^{\prime}(s)\right|\left(\left|w_{t}(s)\right|^{2}+|\nabla w(s)|^{2}+|\Delta u(s)|^{2}+|w(s)|^{2}\right) d x d s \\
& \quad+C \int_{0}^{t}(1+s) \int_{\Omega}\left|b^{\prime}(s)\right|^{2}|\Delta u(s)|^{2} d x d s
\end{aligned}
$$

We note that

$$
\begin{aligned}
\{\sqrt{1+t} w\}_{t t}-b(t) \Delta\{\sqrt{1+t} w\}= & -\frac{1}{4}(1+t)^{-3 / 2} w+(1+t)^{-1 / 2} w_{t} \\
& -\sqrt{1+t} a(x) w_{t}+\sqrt{1+t} b^{\prime}(t) \Delta u(t) .
\end{aligned}
$$

So we can apply (4.14) to $\sqrt{1+s} w$ in place of $u$, then we have for $t \geq T$,

$$
\begin{aligned}
C \int_{T / 4}^{t-T / 4} & (1+s) \int_{\Gamma_{1}}\left|\frac{\partial w}{\partial \tau}\right|^{2} d \Gamma d s \\
\leq & C \int_{0}^{t}(1+s)\left\{\int_{\Gamma_{1}}\left[\left|\frac{\partial w}{\partial v}\right|^{2}+\left|w_{t}(s)\right|^{2}\right] d \Gamma+\int_{\Omega_{L}} a(x)\left|w_{t}(s)\right|^{2} d x\right\} d s \\
& +C \int_{0}^{t}(1+s) \int_{\Omega_{L}}|w(s)|^{2} d x d s+C \int_{0}^{t} \int_{\Omega_{L}}\left(|w(s)|^{2}+\left|w_{t}(s)\right|^{2}\right) d x d s \\
& +C \int_{0}^{t}(1+s) \int_{\Omega}\left|b^{\prime}(s)\right|^{2}|\Delta u(s)|^{2} d s+\frac{\varepsilon_{1}}{4} \int_{0}^{t}(1+s) E_{1}(s) d s .
\end{aligned}
$$

From the equivalence relation of $X_{1}(t)$ and $E_{1}(t)+\|w(t)\|^{2}$, if $n T \leq t<$ $(n+1) T$, we see

$$
\begin{aligned}
\sum_{i=1}^{n} T X_{1}(t-i T) & \leq \sum_{i=0}^{n-1} T X_{1}(i T) \leq C \sum_{i=0}^{n-1} T\left(E_{1}(i T)+E(i T)\right) \\
& \leq C \int_{0}^{t}\left(E_{1}(s)+E(s)\right) d s \leq C\left(X(0)+X_{1}(0)+q(K, E(0))\right)
\end{aligned}
$$

Combining the estimates (5.16)-(5.20) and considering again the argument obtaining (4.17), we have for $t \geq T$, 
$(5.21)$

$$
\begin{aligned}
& (1+t) X_{1}(t)+\tilde{\varepsilon}_{1} \int_{0}^{t}(1+s) E_{1}(s) d s \\
& \leq C\left(X_{1}(0)+E_{1}(0)+X(0)\right)+q(K, E(0)) \\
& \quad+C \int_{0}^{t}(1+s) \int_{\Omega}\left|b^{\prime}(s)\right|\left(\left|w_{t}(s)\right|^{2}+|\nabla w(s)|^{2}+|\Delta u(s)|^{2}+|w(s)|^{2}\right) d x d s \\
& \quad+C \int_{0}^{t}(1+s) \int_{\Omega}\left|b^{\prime}(s)\right|^{2}|\Delta u(s)|^{2} d x d s \quad \text { for all } t>0 .
\end{aligned}
$$

Now, assumption (5.8) and the result (5.13) imply

$$
\begin{aligned}
\int_{0}^{t}(1+ & s) \int_{\Omega}\left|b^{\prime}(s)\right|\left(\left|w_{t}(s)\right|^{2}+|\nabla w(s)|^{2}+|\Delta u(s)|^{2}+|w(s)|^{2}\right) d x d s \\
& +C \int_{0}^{t}(1+s) \int_{\Omega}\left|b^{\prime}(s)\right|^{2}|\Delta u(s)|^{2} d x d s \\
\leq & C\left[K^{3} E(0)^{1 / 2}+K^{4} E(0)+K E(0)^{1 / 2} q_{0}\left(K, E_{1}(0), E(0)\right)\right] .
\end{aligned}
$$

Thus, we have

$$
\begin{aligned}
(1+t) X_{1}(t)+\tilde{\varepsilon}_{1} \int_{0}^{t}(1+s) E_{1}(s) d s \\
\leq C\left[X_{1}(0)+E_{1}(0)+X(0)+q(K, E(0))\right. \\
\left.\quad+K E(0)^{1 / 2} q_{0}\left(K, E_{1}(0), E(0)\right)\right]
\end{aligned}
$$

for all $t>0$.

Now, we can obtain the decay of $E_{1}(t)$, which is the final step of a priori estimates.

Proposition 5.3. We have under the assumption (5.8)

$$
\begin{aligned}
E_{1}(t) \leq C & {\left[X_{1}(0)+E_{1}(0)+X(0)+K^{4} X(0)^{1 / 2}+K^{4} X(0)\right.} \\
& \left.+q(K, E(0))+K E(0)^{1 / 2} q_{0}\left(K, E_{1}(0), E(0)\right)\right](1+t)^{-2} .
\end{aligned}
$$

Proof. By (5.2) we have

$$
\begin{aligned}
\frac{d}{d t}\left[(1+t)^{2} E_{1}(t)\right]= & (1+t) E_{1}(t)+(1+t)^{2} \frac{d}{d t} E_{1}(t) \\
\leq & (1+t) E_{1}(t) \\
& +C(1+t)^{2} \int_{\Omega}\left|b^{\prime}(s)\right|\left(|\nabla w(s)|^{2}+|\Delta u(s)|\left|w_{t}(s)\right|\right) d x
\end{aligned}
$$


The integrability of $(1+t) E_{1}(t)$ is already proved (see (5.23)). Thus from (4.17), (5.8) and (5.11), we have

$$
\begin{aligned}
\int_{0}^{\tilde{T}}(1 & +t)^{2} \int_{\Omega}\left|b^{\prime}(s)\right|\left(|\nabla w(s)|^{2}+|\Delta u(s)|\left|w_{t}(s)\right|\right) d x d t \\
& \leq C K^{2} X(0)^{1 / 2}\left[X_{1}(0)+q(K, E(0))\right]^{1 / 2}+C K^{4} X(0)^{1 / 2} \\
& \leq C\left\{K^{4} X(0)+X_{1}(0)+q(K, E(0))+K^{4} X(0)^{1 / 2}\right\} .
\end{aligned}
$$

Thus, integrating (5.25), then (5.23) and (5.26) imply (5.24).

\section{Completion of the proof of Theorem 2.1}

Set

$$
\begin{aligned}
Q\left(K, E_{1}(0), X(0)\right)=C\{ & X(0)+E_{1}(0)+X_{1}(0)+K^{4} X(0)^{1 / 2}+K^{4} X(0) \\
& \left.+q(K, E(0))+\left(K E(0)^{1 / 2}+1\right) q_{0}\left(K, E_{1}(0), E(0)\right)\right\}
\end{aligned}
$$

and

$$
S_{K} \equiv\left\{\left(u_{0}, u_{1}\right) \in V_{1} \cap\left(H_{2} \times H_{1}\right) \mid Q\left(K, E_{1}(0), X(0)\right)<K^{2}\right\} .
$$

Note that if $K>C\left(\left\|u_{0}\right\|_{H_{2}}+\left\|u_{1}\right\|_{H_{1}}\right)$ and $\left\|u_{0}\right\|_{H_{1}}+\left\|u_{1}\right\|$ is sufficiently small, then $\left(u_{0}, u_{1}\right) \in S_{K}$. Assume that $\left(u_{0}, u_{1}\right) \in S_{K}$, then we get

$$
\left\|u_{t t}(t)\right\|^{2}+\left\|\nabla u_{t}(t)\right\|^{2} \leq Q(1+t)^{-2}<K^{2}(1+t)^{-2}
$$

and

$$
\begin{aligned}
\|\Delta u(t)\|^{2} & \leq\left\|\left(1+\|\nabla u(t)\|^{2}\right) \Delta u(t)\right\|^{2} \\
& \leq\left[\left\|u_{t t}(t)\right\|^{2}+\left\|a(x) u_{t}(t)\right\|^{2}\right] \\
& \leq Q(1+t)^{-2}+C X(0)(1+t)^{-1} \\
& <K^{2}(1+t)^{-1} .
\end{aligned}
$$

Moreover, we have

$$
\begin{aligned}
\int_{0}^{\tilde{T}}\|\Delta u(t)\|^{2} d t & \leq \int_{0}^{\tilde{T}}\left\|\left(1+\|\nabla u(t)\|^{2}\right) \Delta u(t)\right\|^{2} d t \\
& \leq \int_{0}^{\tilde{T}}\left[\left\|u_{t t}(t)\right\|^{2}+\left\|a(x) u_{t}(t)\right\|^{2}\right] d t \\
& \leq \int_{0}^{\tilde{T}} E_{1}(t) d t+C \int_{0}^{\tilde{T}}\left\|u_{t}(t)\right\|^{2} d t \\
& \leq C\left(X_{1}(0)+E_{1}(0)+q(K, E(0))+C q_{0}\left(K, E_{1}(0), E(0)\right)\right. \\
& \leq Q<K^{2}
\end{aligned}
$$


Finally, we note that

$$
\|u(t)\| \leq\left\|u_{0}\right\|+\int_{0}^{t}\left\|u_{t}(s)\right\| d s<\infty, \quad 0<t<\infty .
$$

These estimates show that the assumption (5.8) is true as long as this solution exists and the local solution $u(t)$ can be continued, in fact, on the half interval $[0, \infty)$. This completes the proof of Theorem 2.1.

\section{Proof of Theorem 2.2}

In this section, we consider the problem (1.3). The existence and uniqueness of local solution $u(t)$ of $(1.3)$ in $X_{2}(\tilde{T})$ for $\left(u_{0}, u_{1}\right) \in V_{1}$ is standard, see Lasiecka and Ong [1]. Here, we derive a basic differential inequality for $u(t)$. First, we have the following energy identity

$$
\frac{d}{d t} E(t)+\int_{\Gamma_{1}} b(t) g\left(u_{t}(t)\right) u_{t}(t) d \Gamma+\int_{\Omega} \rho\left(x, u_{t}(t)\right) u_{t}(t) d x=0 .
$$

Now, we differentiate the problem (1.3) with respect to $t$ to linearize it, then $w=u_{t}$ satisfies the following equation;

$$
\begin{gathered}
w_{t t}-b(t) \Delta w-b^{\prime}(t) \Delta u+\rho_{v}(x, w) w_{t}=0 \quad \text { in } \Omega \times(0, \infty), \\
w=0 \quad \text { on } \Gamma_{0} \times(0, \infty), \\
\frac{\partial w}{\partial v}=-g^{\prime}\left(u_{t}\right) w_{t} \quad \text { on } \Gamma_{1} \times(0, \infty), \\
w(0)=u_{1}, \quad w_{t}(0)=b(0) \Delta u_{0}-\rho\left(x, u_{1}\right)=w_{1} \quad \text { in } \Omega .
\end{gathered}
$$

Moreover, the following energy identity holds:

$$
\begin{gathered}
\frac{d}{d t} E_{1}(t)+\int_{\Gamma_{1}} b(t) g^{\prime}\left(u_{t}(t)\right)\left|w_{t}(t)\right|^{2} d \Gamma+\int_{\Omega} \rho_{v}(x, w(t))\left|w_{t}(t)\right|^{2} d x \\
=\frac{1}{2} \int_{\Omega} b^{\prime}(t)|\nabla w(t)|^{2} d x+\int_{\Omega} b^{\prime}(t) \Delta u(t) w_{t}(t) d x .
\end{gathered}
$$

Note that since $\rho_{v} \leq k_{1}<\infty$ for all $t \geq 0$ and $\rho_{v} \geq \varepsilon_{0}$ on $B_{L}^{c} \cup \omega$, it is not different particularly up to the estimates (5.7), we have only to replace $a(x)$ by $\rho_{v}$. Thus we consider the argument proving (5.7) repeatedly, then

$$
\begin{aligned}
X_{1}(t)+ & \varepsilon_{1} \int_{0}^{t} E_{1}(s) d s \\
\leq & C X_{1}(0)+C \int_{0}^{t} \int_{\Omega}\left|b^{\prime}(s)\right|\left(\left|w_{t}(s)\right|^{2}+|\nabla w(s)|^{2}+|\Delta u(s)||w(s)|\right) d x d s \\
& +C \int_{0}^{t} \int_{\Omega}\left|b^{\prime}(s)\right|^{2}|\Delta u(s)|^{2} d x d s
\end{aligned}
$$


where

$$
\begin{aligned}
X_{1}(t)= & \int_{\Omega} w_{t}(t)\left[\phi\left(\left|x-x_{0}\right|\right)\left(x-x_{0}\right)-C_{0} h\right] \cdot \nabla w(t) d x \\
& +\int_{\Omega}(\alpha+\eta) w_{t}(t) w(t) d x+\int_{\Omega} \int_{0}^{w}(\alpha+\eta) \rho_{v}(x, \xi) \xi d \xi d x+k E_{1}(t) .
\end{aligned}
$$

Now, consider the integrals in the right hand side of (6.4). Let $\varepsilon_{2}>0, K>0$ and we assume for a moment

$$
\begin{aligned}
& E(t) \leq \varepsilon_{2}, \quad\left\|u_{t t}(t)\right\|^{2}+\left\|\nabla u_{t}(t)\right\|^{2} \leq K(1+t)^{-1} \\
& \int_{0}^{t}\|\Delta u(s)\|^{2} d s \leq K \quad \text { and } \quad \int_{0}^{t}\left\|\nabla u_{t}(s)\right\|^{2} d s \leq K .
\end{aligned}
$$

Then we have the following Proposition.

Proposition 6.1. Under the assumption (6.5), we have

$$
\begin{gathered}
\int_{0}^{\tilde{T}} \int_{\Omega}\left|b^{\prime}(s)\right|\left(\left|w_{t}(s)\right|^{2}+|\nabla w(s)|^{2}+|\Delta u(s)||w(s)|+\left|b^{\prime}(s)\right||\Delta u(s)|^{2}\right) d x d s \\
\leq C\left[K^{3 / 2} E(0)^{1 / 2}+K E(0)+K^{2} E(0)\right] \equiv q(K, E(0)) \\
X_{1}(t)+\int_{0}^{\tilde{T}} E_{1}(s) d s \leq C X_{1}(0)+C E_{1}(0)+q(K, E(0)) \\
k \int_{0}^{\tilde{T}} \int_{\Omega} \rho_{v}(x, w(t))\left|w_{t}(s)\right|^{2} d x d s+k \int_{0}^{\tilde{T}} \int_{\Gamma_{1}} b(s)\left|w_{t}(s)\right|^{2} d \Gamma d s \\
\leq C X_{1}(0)+C E_{1}(0)+q(K, E(0)) \\
\int_{0}^{\tilde{T}}\|w(t)\|^{2} d t \leq \frac{1}{\varepsilon_{0}} E(0)+C X_{1}(0)+C E_{1}(0)+q(K, E(0)) \\
\equiv q_{0}\left(K, E_{1}(0), E(0)\right) .
\end{gathered}
$$

As section 5, to complete the proof of Theorem 2.2, we set

$$
\begin{aligned}
Q\left(K, E_{1}(0), X(0)\right)=C & \left\{(0)+E_{1}(0)+X_{1}(0)+K E(0)^{2}+q(K, E(0))\right. \\
& \left.+k_{1} q_{0}\left(K, E_{1}(0), E(0)\right)\right\}
\end{aligned}
$$

and

$$
S_{K} \equiv\left\{\left(u_{0}, u_{1}\right) \in V_{1} \cap\left(H_{2} \times H_{1}\right) \mid Q\left(K, E_{1}(0), X(0)\right)<K\right\} .
$$

Assume that $\left(u_{0}, u_{1}\right) \in S_{K}$. A usual computation gives 


$$
\begin{aligned}
\int_{\Omega} b^{\prime}(t) \Delta u(t) w_{t}(t) d x= & -\frac{1}{4} \frac{d}{d t}\left[b^{\prime}(t)\right]^{2}+b^{\prime}(t) \int_{\Omega}|\nabla w(t)|^{2} d x \\
& -b^{\prime}(t) \int_{\Gamma_{1}} g\left(u_{t}(t)\right) w_{t}(t) d \Gamma
\end{aligned}
$$

Combining the above estimates, (6.3) and the condition on $g$, we have

$$
\begin{aligned}
\frac{d}{d t} \tilde{E}_{1}(t) & +\int_{\Gamma_{1}} b(t) g^{\prime}\left(u_{t}(t)\right)\left|w_{t}(t)\right|^{2} d \Gamma+\int_{\Omega} \rho_{v}(x, w(t))\left|w_{t}(t)\right|^{2} d x \\
= & \frac{3}{2} b^{\prime}(t) \int_{\Omega}|\nabla w(t)|^{2} d x-b^{\prime}(t) \int_{\Gamma_{1}} g\left(u_{t}(t)\right) w_{t}(t) d \Gamma \\
\leq & \frac{3}{2} b^{\prime}(t) \int_{\Omega}|\nabla w(t)|^{2} d x+C_{\varepsilon}\left|b^{\prime}(t)\right|^{2} \int_{\Gamma_{1}}\left|u_{t}(t)\right|^{2} d \Gamma+\varepsilon \int_{\Gamma_{1}}\left|w_{t}(t)\right|^{2} d \Gamma
\end{aligned}
$$

where $\tilde{E}_{1}(t)=E_{1}(t)+\left[b^{\prime}(t)\right]^{2} / 4$. Thus the condition on $g$ yields

$$
\begin{gathered}
\frac{d}{d t} \tilde{E}_{1}(t)+\int_{\Gamma_{1}} b(t) g^{\prime}\left(u_{t}(t)\right)\left|w_{t}(t)\right|^{2} d \Gamma+\int_{\Omega} \rho_{v}(x, w(t))\left|w_{t}(t)\right|^{2} d x \\
\leq C\left[\left|b^{\prime}(t)\right| \int_{\Omega}|\nabla w(t)|^{2} d x+\left|b^{\prime}(t)\right|^{2} \int_{\Gamma_{1}}\left|u_{t}(t)\right|^{2} d \Gamma\right] .
\end{gathered}
$$

Hence, from (6.10) we have

$$
\begin{aligned}
& \frac{d}{d t}\left[(1+t) \tilde{E}_{1}(t)\right]=\tilde{E}_{1}(t)+(1+t) \frac{d}{d t} \tilde{E}_{1}(t) \\
& \quad \leq \tilde{E}_{1}(t)+C(1+t)\left[\left|b^{\prime}(t)\right| \int_{\Omega}|\nabla w(t)|^{2} d x+\left|b^{\prime}(t)\right|^{2} \int_{\Gamma_{1}}\left|u_{t}(t)\right|^{2} d \Gamma\right]
\end{aligned}
$$

Now, the estimate (6.7) and assumption (6.5) imply

$$
\begin{aligned}
\int_{0}^{t} \tilde{E}_{1}(s) d s & =\int_{0}^{t}\left[E_{1}(s)+\frac{1}{4}\left[b^{\prime}(s)\right]^{2}\right] d s \\
& \leq C X_{1}(0)+C E_{1}(0)+q(K, E(0))+C K E(0) .
\end{aligned}
$$

Now, we have to derive the integrability of the second term of right hand side of (6.11). Note that from the boundary condition, we have

$$
\begin{aligned}
\left|b^{\prime}(t)\right| & =\left|2\left(\nabla u(t), \nabla u_{t}(t)\right)\right|=2\left|-\left(\Delta u(t), u_{t}(t)\right)+\int_{\Gamma_{1}} \frac{\partial u}{\partial v} u_{t}(t) d \Gamma\right| \\
& \leq 2\|\Delta u(t)\|\left\|u_{t}(t)\right\|+2\left|\int_{\Gamma_{1}} g\left(u_{t}(t)\right) u_{t}(t) d \Gamma\right| \\
& \leq 2\|\Delta u(t)\|\left\|u_{t}(t)\right\|+2 M \int_{\Gamma_{1}}\left|u_{t}(t)\right|^{2} d \Gamma
\end{aligned}
$$


From the above estimate (6.13), (6.1) and the assumption (6.5), we have

$$
\begin{aligned}
\int_{0}^{t}(1+ & s) \int_{\Omega}\left|b^{\prime}(s)\right||\nabla w(s)|^{2} d x d s \\
& \leq C \int_{0}^{t}(1+s)\left[\|\Delta u(s)\|\left\|u_{t}(s)\right\|+\int_{\Gamma_{1}}\left|u_{t}(s)\right|^{2} d \Gamma\right]\|\nabla w(s)\|^{2} d s \\
& \leq C\left[K^{3 / 2} q_{0}\left(K, E_{1}(0), E(0)\right)^{1 / 2}+K E(0)\right]
\end{aligned}
$$

Similarly, (6.1) and assumption (6.5) imply

$$
\begin{aligned}
\int_{0}^{t}(1+ & s)\left|b^{\prime}(s)\right|^{2} \int_{\Gamma_{1}}\left|u_{t}(s)\right|^{2} d \Gamma d s \\
& \leq \int_{0}^{t}(1+s)\|\nabla u(s)\|^{2}\left\|\nabla u_{t}(s)\right\|^{2} \int_{\Gamma_{1}}\left|u_{t}(s)\right|^{2} d \Gamma d s \\
& \leq K E(0)^{2} .
\end{aligned}
$$

Using the above facts (6.14), (6.15) and (6.12), we get

$$
\begin{aligned}
(1+t) E_{1}(t) \leq(1+t) \tilde{E}_{1}(t) \leq & C\left[X_{1}(0)+E_{1}(0)+q(K, E(0))+K E(0)^{2}\right. \\
& \left.+K^{3 / 2} q_{0}\left(K, E_{1}(0), E(0)\right)^{1 / 2}\right] \\
\leq & Q<K
\end{aligned}
$$

where we have used the fact $\tilde{E}_{1}(0)=E_{1}(0)+\left[b^{\prime}(0)\right]^{2} / 4 \leq E_{1}(0)+C K E(0) \leq$ $C q(K, E(0))$. Moreover, we get

$$
\begin{aligned}
\int_{0}^{\tilde{T}}\|\Delta u(s)\|^{2} d s & \leq \int_{0}^{\tilde{T}}\left[\left\|u_{t t}(t)\right\|^{2}+\left\|\rho\left(x, u_{t}(t)\right)\right\|^{2}\right] d t \\
& \leq \int_{0}^{\tilde{T}} E_{1}(t) d t+k_{1} \int_{0}^{\tilde{T}}\left\|u_{t}(t)\right\|^{2} d t \\
& \leq C\left[X_{1}(0)+E_{1}(0)+q(K, E(0))\right]+k_{1} q_{0}\left(K, E_{1}(0), E(0)\right) \\
& \leq Q<K
\end{aligned}
$$

Also, we can obtain

$$
\int_{0}^{\tilde{T}}\|\nabla w(s)\|^{2} d s \leq \int_{0}^{\tilde{T}} E_{1}(s) d s \leq Q<K .
$$

Finally, $\|u(t)\|$ is estimated as usual. Thus as section 5, we conclude that the local solution $u(t)$ can be continued on the half interval $[0, \infty)$. This completes the proof of Theorem 2.2. 


\title{
References
}

[1] Lasiecka, I. and Ong, J., Global solvability and uniform decays of solutions to quasilinear equation with nonlinear boundary dissipation, Comm. Partial Diff. Eqs., 24 (1999), 20692107.

[2] Lasiecka, I. and Triggiani, R., Uniform stabilization of the wave equation with Dirichlet or Neumann feedback control without geometric conditions, Appl. Math. Optmiz., 25 (1992), 189-224.

[3] Lions, J. L., Exact contorollability, stabilization and perturbation for distributed systems, SIAM Rev., 30 (1988), 1-68.

[4] Mochizuki, K., Global existence and energy decay of small solutions to the Kirchhoff equation with linear dissipation localized near infinity, J. Math. Kyoto Univ., 39 (1999), 347363.

[5] Nakao, M., Energy decay for the linear and semilinear wave equations in exterior domains with some localized dissipation, Math. Z., 238 (2001), 781-797.

[6] Nakao, M. and Bae, J. J., Existence of global solutions to the Cauchy problem of Kirchhoff type quasilinear wave equation with weakly nonlinear dissipation, Funk. Ekvac., 45 (2002), 387-395.

[ 7 ] Bae, J. J. and Nakao, M., Energy decay for the wave equation with boundary and localized dissipations in exterior domains, Math. Nach., to appear.

[ 8 ] Bae, J. J. and Nakao, M., Existence problem for the Kirchhoff type equation with a localized weakly nonlinear dissipation in exterior domains, Dis. Conti. Dyn. Sys., 11 (2004), 731-743.

[9] Russell, D. L., Exact boundary value controllability theorems for wave and heat processes in star-comple-mented regions in Differential Games and Control Theory, Roxin, Liu and Sternberg, Eds. Marcel Dekker Inc., New York, 1974.

[10] Yamada, Y., On some quasilinear wave equation with dissipative terms, Nagoya Math. J., 187 (1982), 17-39.

\author{
nuna adreso: \\ Department of Mathematics \\ University of Ulsan \\ Ulsan 680-749 \\ Korea \\ E-mail: jjbae@pusan.ac.kr
}

(Ricevita la 28-an de aŭgusto, 2003)

(Reviziita la 15-an de septembro, 2003) 